University of Wollongong

Research Online

Faculty of Engineering and Information

Faculty of Engineering and Information

Sciences - Papers: Part B

Sciences

2018

\title{
Magnetically confined electron beam system for high resolution electron transmission-beam experiments
}

\author{
A I. Lozano \\ Consejo Superior De Investigaciones Científicas \\ J C. Oller \\ Centro De Investigaciones Energticas, Medioambientales Y Tecnlogicas \\ K Krupa \\ Universidade Nova de Lisboa \\ F Ferreira da Silva \\ Universidade Nova de Lisboa \\ Paulo Limao-Vieira \\ Universidade Nova de Lisboa
}

See next page for additional authors

Follow this and additional works at: https://ro.uow.edu.au/eispapers1

Part of the Engineering Commons, and the Science and Technology Studies Commons

Research Online is the open access institutional repository for the University of Wollongong. For further information contact the UOW Library: research-pubs@uow.edu.au 


\title{
Magnetically confined electron beam system for high resolution electron transmission-beam experiments
}

\author{
Abstract \\ A novel experimental setup has been implemented to provide accurate electron scattering cross sections \\ from molecules at low and intermediate impact energies (1-300 eV) by measuring the attenuation of a \\ magnetically confined linear electron beam from a molecular target. High-resolution electron energy is \\ achieved through confinement in a magnetic gas trap where electrons are cooled by successive collisions \\ with $\mathrm{N}_{2}$. Additionally, we developed and present a method to correct systematic errors arising from \\ energy and angular resolution limitations. The accuracy of the entire measurement procedure is validated \\ by comparing the $\mathrm{N}_{2}$ total scattering cross section in the considered energy range with benchmark values \\ available in the literature. \\ Disciplines \\ Engineering | Science and Technology Studies

\section{Publication Details} \\ Lozano, A. I., Oller, J. C., Krupa, K., Ferreira da Silva, F., Limao-Vieira, P., Blanco, F., Munoz, A., Colmenares, \\ R. \& Garcia, G. (2018). Magnetically confined electron beam system for high resolution electron \\ transmission-beam experiments. Review of Scientific Instruments, 89 (6), 063105-1-063105-7.

\section{Authors} \\ A I. Lozano, J C. Oller, K Krupa, F Ferreira da Silva, Paulo Limao-Vieira, Francisco Blanco, Antonio Munoz, R \\ Colmenares, and Gustavo Garcia
}




\title{
Magnetically confined electron beam system for high resolution electron transmission-beam experiments
}

\author{
A. I. Lozano, ${ }^{1, a}$ ) J. C. Oller, ${ }^{2}$ K. Krupa, ${ }^{1,3}$ F. Ferreira da Silva, ${ }^{3}$ P. Limão-Vieira, ${ }^{3}$ F. Blanco, ${ }^{4}$ \\ A. Muñoz, ${ }^{2}$ R. Colmenares, ${ }^{5}$ and G. García ${ }^{1,6, b)}$ \\ ${ }^{1}$ Instituto de Física Fundamental, Consejo Superior de Investigaciones Científicas, Madrid, Spain \\ ${ }^{2}$ Centro de Investigaciones Energéticas Medioambientales y Tecnológicas, Madrid, Spain \\ ${ }^{3}$ Laboratório de Colisões Atómicas e Moleculares, CEFITEC, Departamento de Física, \\ Universidade NOVA de Lisboa, 2829-516 Caparica, Portugal \\ ${ }^{4}$ Departamento de Física Atómica, Molecular y Nuclear, Universidad Complutense de Madrid, Madrid, Spain \\ ${ }^{5}$ Servicio de Radiofísica, Hospital Universitario Ramón y Cajal, Madrid, Spain \\ ${ }^{6}$ Centre of Medical Radiation Physics, University of Wollongong, NSW, Australia
}

(Received 19 March 2018; accepted 18 May 2018; published online 6 June 2018)

\begin{abstract}
A novel experimental setup has been implemented to provide accurate electron scattering cross sections from molecules at low and intermediate impact energies (1-300 eV) by measuring the attenuation of a magnetically confined linear electron beam from a molecular target. High-resolution electron energy is achieved through confinement in a magnetic gas trap where electrons are cooled by successive collisions with $\mathrm{N}_{2}$. Additionally, we developed and present a method to correct systematic errors arising from energy and angular resolution limitations. The accuracy of the entire measurement procedure is validated by comparing the $\mathrm{N}_{2}$ total scattering cross section in the considered energy range with benchmark values available in the literature. Published by AIP Publishing. https://doi.org/10.1063/1.5030068
\end{abstract}

\section{INTRODUCTION}

Magnetically confined beam techniques for positron scattering experiments were implemented by Surko et al. ${ }^{1,2}$ where a buffer gas trap in a magnetic field stores and cools positrons. In such a device, a pulsed positron beam was produced and accelerated toward a scattering chamber. Further development of the Surko's trap principles can be found elsewhere in different experimental setups. ${ }^{3,4}$ In particular, the state-of-theart positron beam lines available at the Australian National University $^{5,6}$ are clear examples of how these experimental systems can be used for both fundamental scattering studies and material science analysis. A recent review on trap-based techniques and their applications to positron beams has been published by Danielson et al. ${ }^{7}$ Recently we have explored the working principle of Surko's trap to the particular case of electron beams and the methodology to implement it. A literature survey has shown, as far as the authors are aware, that the experimental arrangement reported here is the first electron trap combined with a pulsed beam-transmission technique to obtain accurate electron scattering cross sections from key selected molecular targets. Back in 2013, we have designed a first prototype based on a continuous electron beam confined by an intense axial magnetic field passing through a gas cell. ${ }^{8,9}$ More recently, this setup has been improved in terms of energy and angular resolution by incorporating a pulsing beam system and more efficient data collection and analysis procedures. $^{10}$

In this paper, we present an improved version of the previous experimental system that incorporates a nitrogen trap to

\footnotetext{
a) anita_ilm@iff.csic.es

b)g.garcia@csic.es
}

cool an electron beam produced by a hairpin filament to an energy resolution as good as $100 \mathrm{meV}$. In addition, by using nitrogen as a buffer gas in the trap, the emitting filament is now insulated from potentially reactive target molecules that could migrate to its surface affecting the filament's emission efficiency. ${ }^{9}$ The technical details of the experimental setup and its capability to provide accurate electron scattering cross sections and transmission energy spectra are evaluated in this study using nitrogen also as a target molecule. The present results are compared with benchmarked data available in the literature, and the influence of systematic errors associated with the angular resolution and multiple scattering processes are properly evaluated by using differential elastic cross sections obtained through our independent atom model with the screening corrected additivity rule (IAM-SCAR+I) method which includes interference effects. ${ }^{11}$ The present experimental setup is described in detail in Sec. II. The operation conditions and transmitted energy spectra showing the cooling electron procedure are given in Sec. III. Benchmarking measurements for the molecular nitrogen, estimation of the uncertainty limits, and comparison with available theoretical and experimental data are presented in Sec. IV. Finally, concluding remarks are presented in Sec. V.

\section{EXPERIMENTAL METHOD}

\section{A. Setup}

A schematic diagram of the magnetically confined electron beam setup is shown in Fig. 1. The electron beam line is divided into four sections: electron gun (EG), gas trap (GT), scattering chamber (SC), and detector area (AD). The last three sections are mutually separated by differential pumping, 


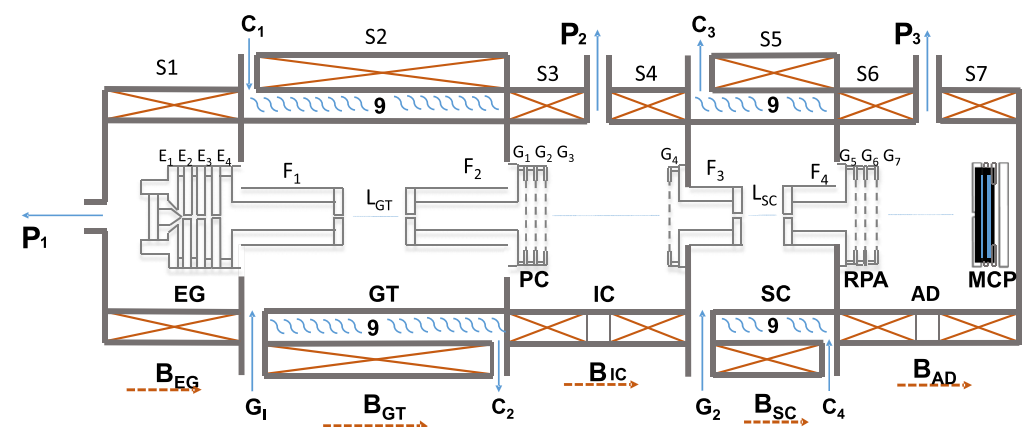

FIG. 1. Schematic diagram of the experimental setup (see text for operation details). and all sections are externally surrounded by solenoids which generate axial magnetic fields of different intensities.

The electron gun consists of a commercial hairpin filament (AGAR A054) whose tip is located between two electrodes (E1) and (E2), the repeller, and the extractor. A negative accelerating voltage (Va) is applied to E1. A third electrode (E3) controls the electron intensity that is transmitted to the gas trap (GT). A forth electrode (E4) is electrically connected to GT fixing the kinetic energy of the electrons entering the trap $\left(\mathrm{V}_{\mathrm{a}}-\mathrm{V}_{\mathrm{GT}}\right)$. The first solenoid (S1) generates a weak magnetic field (typically $50 \mathrm{G}$ ) in the gun area $\left(\mathrm{B}_{\mathrm{EG}}\right)$ just to confine the primary beam extraction. The electron gun vacuum chamber is differentially pumped by an $80 \mathrm{I} / \mathrm{s}$ turbo-pump which provides a background pressure of $10^{-8}$ Torr and allows us to keep it below $10^{-6}$ Torr during the GT operation conditions.

The GT chamber is formed by a $240 \mathrm{~mm}$ length cylinder surrounded by the second solenoid (S2) which is cooled internally by a closed-loop cooling jacket and externally by a cooling fan system. The effective collision length of the trap $\left(\mathrm{L}_{\mathrm{GT}}\right)$ is reduced to $60 \mathrm{~mm}$ by means of $\mathrm{F}_{1}$ and $\mathrm{F}_{2}$ appropriate fittings (see Fig. 1). This length is set by two $1.5 \mathrm{~mm}$ diameter apertures attached to the ends of these fittings and corresponds to the length for which the axial magnetic field $\mathrm{B}_{2}$ (generated by $\mathrm{S}_{2}$ ) can be considered uniform in this region. The trap typically operates with a $\mathrm{N}_{2}$ gas pressure of 60 mTorr (introduced through a needle valve) and an axial magnetic field $\left(\mathrm{B}_{\mathrm{GT}}\right)$ of 0.1 Tesla. The vacuum chamber interface (IC) between the GT and the SC is differentially pumped by a $300 \mathrm{l} / \mathrm{s}$ turbopump maintaining the pressure below $10^{-6}$ Torr during the measurements. This interface chamber is surrounded by two solenoids (S3 and S4) which generate an axial magnetic field $\left(B_{I C}\right)$. Inside this chamber, a three-grid system $\left(G_{1}, G_{2}\right.$, and $\mathrm{G}_{3}$ ), attached to the exit of the GT, is used to pulse the beam by applying a negative squared pulse $\left(-\mathrm{V}_{\mathrm{p}}\right)$ to $\mathrm{G}_{2}$. Additionally, another grid $\left(\mathrm{G}_{4}\right)$ is attached to the SC and electrically connected to the same negative/positive power supply setting the chamber potential $\left(\mathrm{V}_{\mathrm{SC}}\right)$ which decelerates/accelerates the electron beam at the SC entrance. The electron's kinetic energy in the $\mathrm{SC}$ is then set as $\mathrm{V}_{\mathrm{a}}$ minus/plus $\mathrm{V}_{\mathrm{SC}}$, depending on the $\mathrm{V}_{\mathrm{SC}}$ voltage bias.

The SC is formed by a $140 \mathrm{~mm}$ length cylinder and externally surrounded by the fifth solenoid (S5). The molecular target is introduced through a leak valve maintaining a uniform pressure which was varied from 0 to 9 mTorr during the measurements. Using a similar fitting and aperture systems as those for the GT, the collision path length of the $\mathrm{SC}$, being delimited by two $1.5 \mathrm{~mm}$ diameter apertures, is reduced to $40 \mathrm{~mm}$ which is the length where the magnetic field remains uniform (within 2.5\%). The gas pressure is measured in two different points along the SC by means of a calibrated MKS-micro-Pirani gauge and an absolute capacitance MKSBaratron (627B) manometer. The temperature in this chamber is also controlled with a standard K-type thermocouple sensor. The $1.5 \mathrm{~mm}$ diameter exit aperture of the SC is connected with the analyser-detector chamber AD through a three-grid $\left(\mathrm{G}_{5}, \mathrm{G}_{6}\right.$, and $\left.\mathrm{G}_{7}\right)$ system which configures the retarding potential analyser (RPA).

The $\mathrm{AD}$ is a $350 \mathrm{~mm}$ length chamber differentially pumped by a 300 1/s turbo pump. Two solenoids (S6 and S7) provide the axial magnetic field $\left(\mathrm{B}_{\mathrm{AD}}\right)$. The electron detector is a double micro-channel-plate (MCP) system operating in the single pulse counting mode. The pulsing, counting, and data analysis processes are entirely controlled by a custom-made LabVIEW (National Instruments) programme.

\section{B. Applied voltages and magnetic fields}

The experimental configuration of the magnetically confined electron beam system is composed of 5 independent axial magnetic fields generated by the corresponding cylindrical solenoids and their respective current power supplies. The axial magnetic field (B) plays a central role in the present transmission beam measurements by confining the electron beam within a scattering angle $\theta$ (see below). The electron's velocity component which is parallel to the field direction $\left(\mathrm{v}_{\mathrm{II}}\right)$ is not affected by the magnetic force, whereas the perpendicular component $\left(\mathrm{v}_{\perp}\right)$ interacts with the field, and the resulting magnetic force $\left(\mathbf{F}_{\mathrm{B}}\right)$ generates a circular motion which modifies the electron's trajectory. The combination of $v_{I I}$ and $v_{\perp}$ results in a helix of radius $\mathbf{R}_{\mathrm{B}}$ (cycloidal motion) (as schematically shown in Fig. 2) which is given as

$$
\mathbf{R}_{\mathrm{B}}=\frac{\mathrm{m}}{\mathrm{e}} \frac{\mathrm{v}_{\perp}}{\mathbf{B}},
$$

where $\mathrm{m}$ and e are the electron mass and charge.

B does not change the total kinetic energy $\left(E_{K}\right)$, but it is convenient to write

$$
E_{K}=E_{\mathrm{II}}+E_{\perp},
$$

with $E_{\mathrm{II}}$ and $E_{\perp}$ the kinetic energies related to the parallel and perpendicular velocity components, which can be given as

$$
\begin{aligned}
& E_{\mathrm{II}}=E_{\mathrm{k}} \cos ^{2} \theta, \\
& E_{\perp}=E_{\mathrm{k}} \sin ^{2} \theta .
\end{aligned}
$$




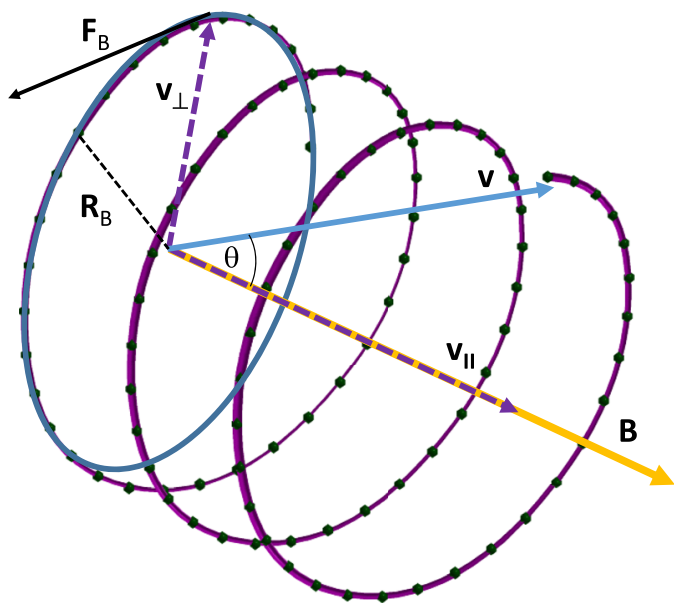

FIG. 2. Electron movement in an axial magnetic field (B).

The working operation conditions are based on applying axial magnetic field magnitudes in the GT and the SC high enough to ensure that $\mathbf{R}_{\mathrm{B}}$, in comparison with the chamber apertures, can be considered $\mathbf{R}_{\mathrm{B}} \cong 0$. Under these conditions, the path length of electrons traveling through the GT and the SC can be considered equal to its respective geometrical length and the effect of any scattered event can be regarded as an $E_{\text {II }}$ energy loss. Of relevance is the fact that scattered electrons for scattering angles $\theta>90^{\circ}$ are axially reversed toward the EG region.

The magnitude of the magnetic field was measured along the axis of the GT and the SC with a Hirst Magnetics GM08 gaussmeter using an axial hall probe ( $1 \%$ accuracy). The corresponding results are shown in Fig. 3, where a "plateau" region is achieved around the centre of each chamber. The effective scattering lengths of the GT and the SC have been adjusted to the length of their respective plateau to ensure that the axial magnetic field inside both the gas chambers $\left(\mathbf{B}_{\mathrm{GT}}\right.$ and $\mathbf{B}_{\mathrm{SC}}$ ) can be considered uniform (within 2.5\%). In the current

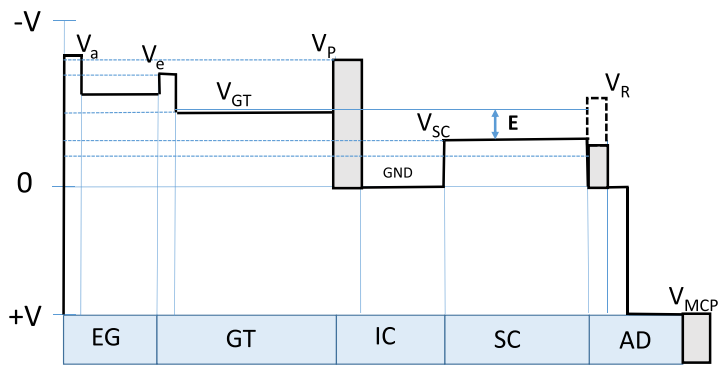

FIG. 4. Applied voltages to the different parts of the electron beam system: electron gun (EG), gas trap (GT), interaction chamber (IC), scattering chamber (SC), and analyser-detector (AD).

configuration, we operate at $\mathbf{B}_{\mathrm{GT}}=\mathbf{B}_{\mathrm{SC}} \cong 0.1 \mathrm{~T}(1000 \mathrm{G})$. The role of the other magnetic fields is simply to focus the electron emission $\left(\mathbf{B}_{\mathrm{EG}}\right)$ and guide the beam between the two main chambers and the final detector $\left(\mathbf{B}_{\mathrm{IC}}, \mathbf{B}_{\mathrm{AD}}\right)$. Their respective magnitudes were tuned to optimize the electron current during the measurements. The schematics of the potentials applied to the different regions of the beam system are shown in Fig. 4.

Electrons emitted by the heated filament are accelerated through the GT with a kinetic energy $\left|V_{a}-V_{G T}\right| \cong 7 \mathrm{eV}$. There, they are confined by means of $V_{p}$ for about $90 \mathrm{~ms}$ losing almost all of their energy by successive collisions with the $\mathrm{N}_{2}$ molecules which are maintained in the chamber at a constant pressure of about 60 mTorr. The kinetic energy of the electron beam inside the GT is tuned to $7 \mathrm{eV}$ where the cross sections of $\mathrm{N}_{2}$ are appreciable ${ }^{12}$ and the transmitted electrons are fast enough to optimize the cooling collision processes. An adjustable positive voltage pulse (0-500 $\mathrm{V}, 0-50 \mathrm{~ms}$ duration) provided by a DEI PVM-4210 pulse generator is applied over $\mathrm{V}_{\mathrm{P}}$ to yield a pulsed electron beam reaching the $\mathrm{SC}$ with a kinetic energy $\mathrm{E}=\mid \mathrm{V}_{\mathrm{GT}}-\mathrm{V}_{\mathrm{SC}} \mathrm{l}$, setting therefore the incident collision energy for electrons scattered from a target molecule. $\mathrm{V}_{\mathrm{a}}$ is typically $-20 \mathrm{~V}$; thus, collision energies above $20 \mathrm{eV}$ are
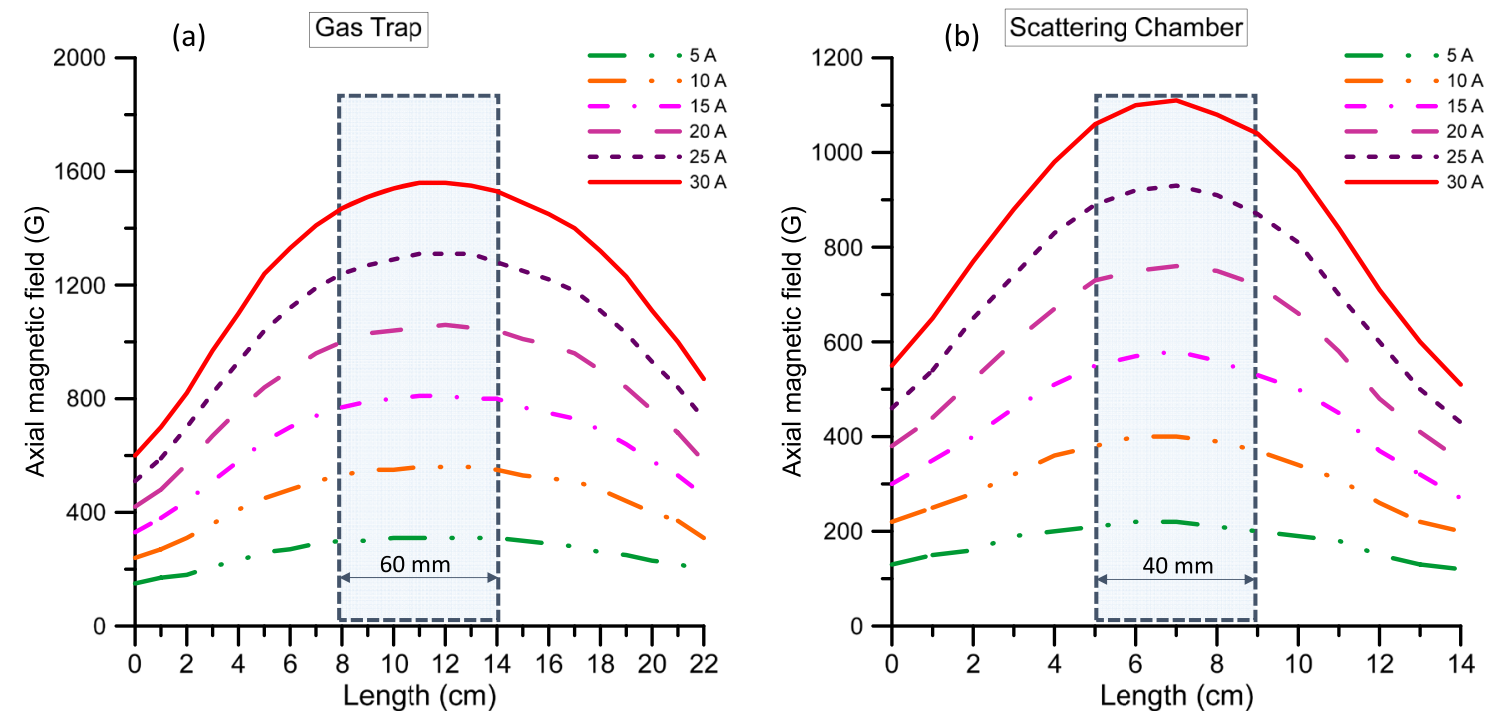

FIG. 3. Magnitude of the axial magnetic field along the GT (a) and the SC (b) as a function of the length for different solenoid currents. The interaction region lengths of 60 and $40 \mathrm{~mm}$ are also shown. 
achieved by applying a $\mathrm{V}_{\mathrm{SC}}$ positive voltage. The maximum kinetic energy that can be reached with this procedure depends on the maximum intensity obtained for the $\mathbf{B}_{\mathrm{SC}}$ magnetic field in order to ensure the aforementioned condition $\mathbf{R}_{\mathrm{B}} \cong 0$. For the present experimental setup, we can estimate a maximum electron energy in the $\mathrm{SC}$ of $300 \mathrm{eV}$. After the scattering chamber, a negative retarding potential $V_{R}$ is swept from 0 to $V_{G T}$ in order to obtain the integrated energy spectra of the transmitted electrons. Finally, electrons passing through the retarding potential barrier are accelerated into the cathode electrode of a double MCP system with a $2000 \mathrm{~V}$ drop biasing between the anode and cathode. The cathode electrode is maintained at $+100 \mathrm{~V}$ in order to avoid secondary electron losses from the cathode.

\section{MEASUREMENT PROCEDURE}

As a combination of the applied voltage through the filament and the thermionic electron emission process, the natural energy spread of the primary electron beam is about $1 \mathrm{eV}$. The beam is confined by the magnetic and electrostatic fields described above in the GT where electrons lose their initial kinetic energy by elastic and inelastic collisions with the $\mathrm{N}_{2}$ molecules maintained in the chamber at a constant pressure of 60 mTorr. The initial energy of electrons entering the GT is about $7 \mathrm{eV}$ in order to optimize the efficiency of the cooling process. After this process, typically $500-900 \mathrm{~ms}$ long electron pulses are formed and accelerated to the SC with a repetition rate of $10 \mathrm{~Hz}$. Typical transmitted electron intensities as a function of the retarding potential for different $\mathrm{N}_{2}$ gas pressure values in the GT are shown in Fig. 5. In this figure, the maximum electron currents are normalized to 100 , and hence, they are comparable for the lower RPA voltages.

Such an experimental arrangement leads to typical electron energy resolutions $(\Delta \mathrm{E})$ in the range of $100-200 \mathrm{meV}$. Note that residual geometrical misalignments between the different chambers contribute to limit the achieved $\Delta \mathrm{E}$ values.
Notwithstanding, such resolution values are enough to accomplish the total electron scattering cross section experiments.

\section{A. Total electron scattering cross sections}

In order to evaluate the reliability of the present experimental system to determine accurate electron scattering cross sections, we have performed some preliminary measurements with molecular nitrogen. $\mathrm{N}_{2}$ was introduced in the SC through a leak valve to maintain a uniform gas pressure inside the chamber in the range 0.1-10 mTorr. Once checked that the energy resolution of the primary beam is within the required $\Delta \mathrm{E}$ range by sweeping the RPA voltage, this voltage is fixed to the operation value $\left(\mathrm{V}_{\mathrm{o}}\right)$ which reduces the transmitted intensity to $25 \%$ of the total primary intensity [see Fig. 5(a)]. This procedure improves notably the "effective" energy resolution for transmission measurements since it increments the capability of the RPA to discriminate the transmitted intensity against scattered electrons. Under these conditions, the pulse controlling and data acquisition software programme alternatively stores the transmitted intensity when the beam pulse is on and off (background for subtraction) for different gas pressures, typically 10 samples. During the measurements, the pressure range is rearranged for each incident energy in order to ensure that multiple scattering effects are negligible. This is accomplished by checking that the attenuation of the transmitted electron intensity in this pressure range follows a single exponential decay (see Fig. 6). The control and data acquisition software described above is also programmed to display the attenuation of the beam and to calculate the slope of the corresponding semi-logarithmic plot as those shown in Fig. 6. This slope provides the total cross section through the well-known Lambert-Beer attenuation law

$$
I=I_{0} e^{-n \sigma_{T} L},
$$

where $I$ is the transmitted electron intensity, $I_{0}$ the initial intensity, $n$ the molecular gas density, $\sigma_{\mathrm{T}}$ the total cross section, and $L$ the interaction path length. By assuming an ideal gas
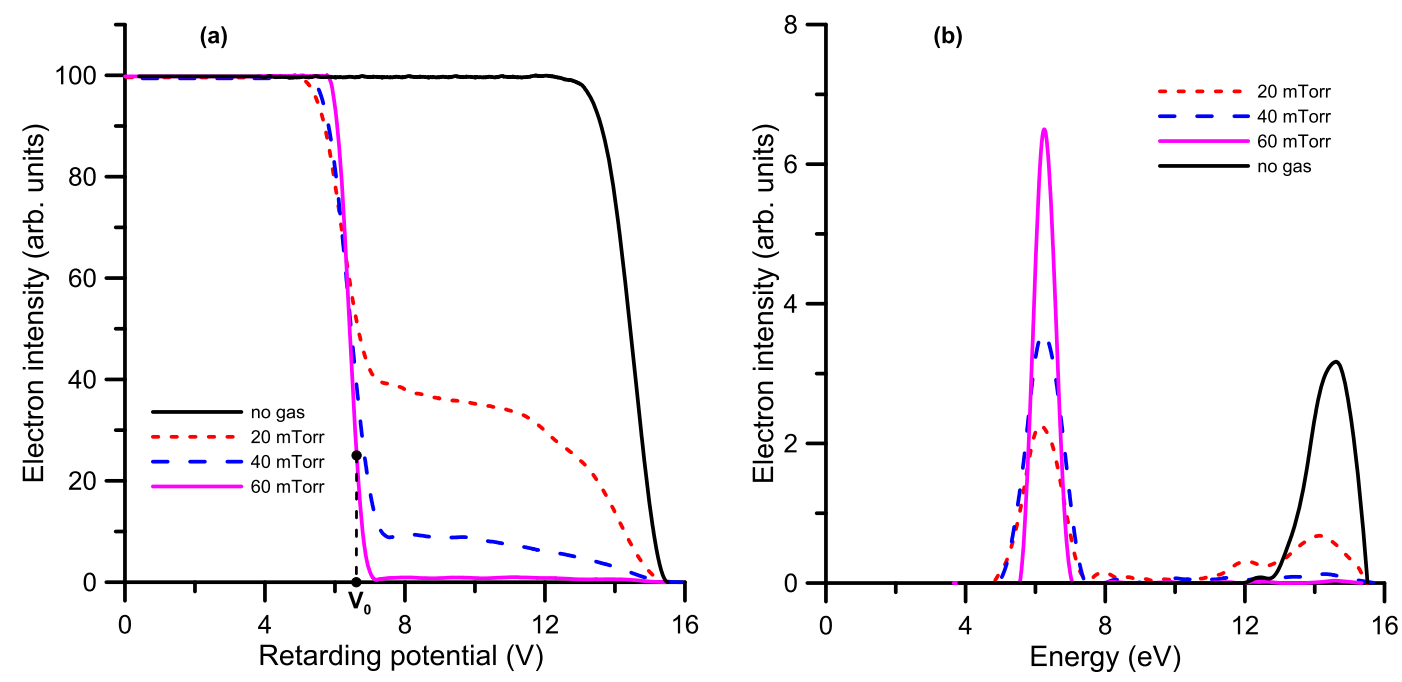

FIG. 5. (a) Transmitted electron intensity as a function of the RPA voltage for different $\mathrm{N}_{2}$ gas pressure values in the GT. •, operation voltage ( $\mathrm{V}_{0}$ ). (b) Energy distribution of transmitted electrons, derivative of the curves shown in (a). 


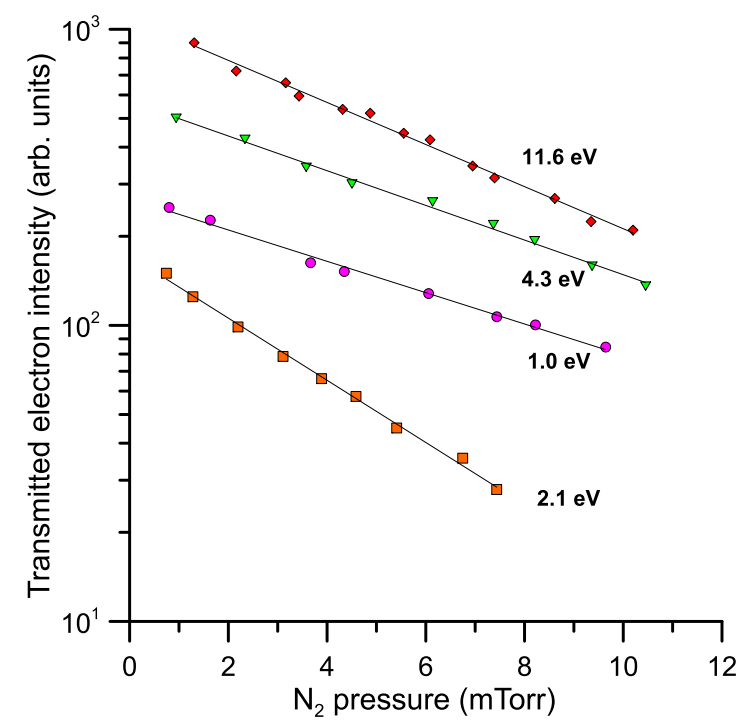

FIG. 6. Transmitted electron intensity as a function of the target $\left(\mathrm{N}_{2}\right)$ pressure in the SC for different incident energies.

behavior, this equation can be written as

$$
\ln \left(\frac{I}{I_{0}}\right)=-\frac{L \sigma_{T}}{k T} p,
$$

where $k$ is the Boltzmann's constant, $T$ is the absolute temperature, and $p$ is the gas pressure. $T$ is derived from $T=\sqrt{T_{c} T_{m}}$, where $T_{c}$ and $T_{m}$ are the temperature of the scattering chamber measured with a thermocouple and the Baratron gauge. According to the above procedure, a semi-logarithmic plot of Eq. (6) as a function of $p$ provides the slope $(m)$ which is related to $\sigma_{\mathrm{T}}$ as

$$
\sigma_{T}=\frac{m k T}{L} .
$$

For each incident electron energy, attenuation measurements were repeated at least 5 times to ensure that statistical uncertainties remained below 4\%. Other random uncertainties are due to temperature measurement (within $1 \%$, according to manufacturer's data) and the numerical fitting procedure (about 1\%). By combining these uncertainties, a total uncertainty limit of $5 \%$ has been determined. Important systematic errors arise from the acceptance (missing) angles of the analyser-detector system, which will be discussed in the next section, and the determination of the actual interaction region length, which will be estimated by a comparison with benchmark data (see Sec. IV).

As described elsewhere, ${ }^{13,14}$ for incident energies below the electronic excitation threshold of the target molecule, differential elastic cross sections can be derived from the integrated transmission spectra provided by the RPA when the target pressure in the $\mathrm{SC}$ is low enough to ensure single scattering conditions.

\section{B. Missing angles}

Due to the confinement effect of the intense axial magnetic field, the angular resolution $(\Delta \theta)$ is dependent on the energy resolution $(\Delta E)$. If an electron with incident energy $E_{K}$ collides with a target molecule, its kinetic energy after the collision $E^{\prime}{ }_{K}$ is given by $E^{\prime}{ }_{K}=E_{K}$ for elastic processes (the kinetic energy transferred from the incident electron to the target molecule is negligible because of their relative mass ratio) and $E^{\prime}{ }_{K}=E_{K}$ $+E_{\text {exc }}$ for inelastic processes, where $E_{\text {exc }}$ is the internal energy transferred to the target molecule during the collision. Again, the energy of the scattered electron can be written as

$$
E_{K}^{\prime}=E_{\mathrm{II}}{ }^{\prime}+E_{\perp}^{\prime}
$$

and according to Eq. (3)

$$
E_{\mathrm{II}}{ }^{\prime}=E_{\mathrm{k}} \cos ^{2} \theta
$$

For an elastic collision, the angular resolution $(\Delta \theta)$ represents the smallest scattering angle for which the detector is able to distinguish between scattered and unscattered electrons. Considering the case of the minimum deflection distinguished by the detector, $\Delta \theta$ can be derived from $\Delta E$ as

$\Delta E=E_{K}-E_{\mathrm{II}}{ }^{\prime}$, which in this case corresponds to $E_{\mathrm{II}^{\prime}}$ $=E \cos ^{2}(\Delta \theta)$; thus,

$\Delta E=E_{K}-E_{K} \cos ^{2}(\Delta \theta)$, resulting in

$$
\Delta \theta=\arccos \sqrt{1-\frac{\Delta E}{E_{K}}} .
$$

Electrons elastically scattered within $\Delta \theta$ and $180^{\circ}-\Delta \theta$ angles are considered by the detector as unscattered, and therefore, the total cross sections measured with the attenuation procedure tend to be lower than the real cross sections. A similar situation is found for the rotational excitation processes. Rotational excitation energies are very low, typically a few meV or tenths of meV, which are clearly lower than $\Delta E$, so the RPA sensitivity is not able to resolve them. Note that dipole interactions are strongly peaked in the forward direction and consequently the $\Delta \theta$ limitation is even more critical for rotational excitation than for elastic scattering processes. The magnitude of this systematic error, $\sigma(\Delta \theta)$, which is inherent to the present measurements, can be evaluated from theoretical data by integrating the calculated differential cross sections (DCS) over the missing experimental angles, which is given as

$$
\begin{aligned}
\sigma(\Delta \theta)= & 2 \pi\left(\int_{0}^{\Delta \theta} \frac{d\left(\sigma_{e l}+\sigma_{r o t}\right)}{d \Omega} \sin \theta d \theta\right. \\
& \left.+\int_{180-\Delta \theta}^{180} \frac{d\left(\sigma_{e l}+\sigma_{r o t}\right)}{d \Omega} \sin \theta d \theta\right),
\end{aligned}
$$

where $\sigma_{e l}$ and $\sigma_{\text {rot }}$ represent the elastic and rotational cross sections. Depending on the target, vibrational excitation energies of the ground state may be lower than $\Delta E$ so contributing to the $\sigma(\Delta \theta)$ term. However, reliable differential vibrational excitation cross sections are scarce, and the available data indicate that their contributions to this systematic error can be neglected, in comparison with the elastic and rotational excitation contributions. As an example, in the case of $\mathrm{N}_{2}$ for energies around $2 \mathrm{eV}$, the experimental inelastic $(0 \rightarrow 1)$ DCS is about one order of magnitude lower than the elastic $(0 \rightarrow 0)$ DCS for relatively small scattering angles $\left(\sim 15^{\circ}\right){ }^{15}$ 


\section{BENCHMARKING MEASUREMENTS}

To check the reliability of the described measurement procedure and to estimate possible systematic errors affecting the experimental results, total electron scattering cross sections for $\mathrm{N}_{2}$ were measured in the energy range 1-300 eV. As described in Sec. III. A, attenuation measurements were performed by varying the $\mathrm{N}_{2}$ pressure in the SC and recording the transmitted electron intensity through the RPA when the retarding potential is tuned to discriminate $75 \%$ of the initial electron intensity (without $\mathrm{N}_{2}$ in the $\mathrm{SC}$ ). Attenuation curves for incident electron energies in the range $1.0-11.6 \mathrm{eV}$ are shown in Fig. 6. The pressure range of measurements has been chosen for each incident electron energy to obtain a significant attenuation of the primary beam (typically up to $20 \%$ of the incident intensity) but ensuring that multiple scattering effects did not contaminate the measurements.

The present experimental total cross sections (TCSs) for electron impact with $\mathrm{N}_{2}$ in the energy range $1-20 \mathrm{eV}$ are plotted in Fig. 7 together with the previous measurements from Szmytkowski and Maciag ${ }^{16}$ and the recommended values from Itikawa. $^{12}$

The total uncertainty related to random sources is estimated to be better than 5\%. However, other important systematic error sources need to be considered in order to provide reliable reference data. The relevance of the missing angle effect described above can be estimated with the aid of theoretical calculations. For this purpose, we have used our independent atom model with screened corrected additivity rule and interference effects (IAM-SCAR+I) method which has been described in detail elsewhere ${ }^{17-19}$ For an electron energy resolution of $200 \mathrm{meV}$, the missing angles given by Eq. (9) range from $6^{\circ}$ to $29^{\circ}$ for incident energies from 1 to $300 \mathrm{eV}$. From Fig. 7, the total cross section calculated with the IAM-SCAR+I procedure agrees very well with the present measurements for energies above $15 \mathrm{eV}$. Given that the calculation methodology considers only geometrical properties of the molecule and not

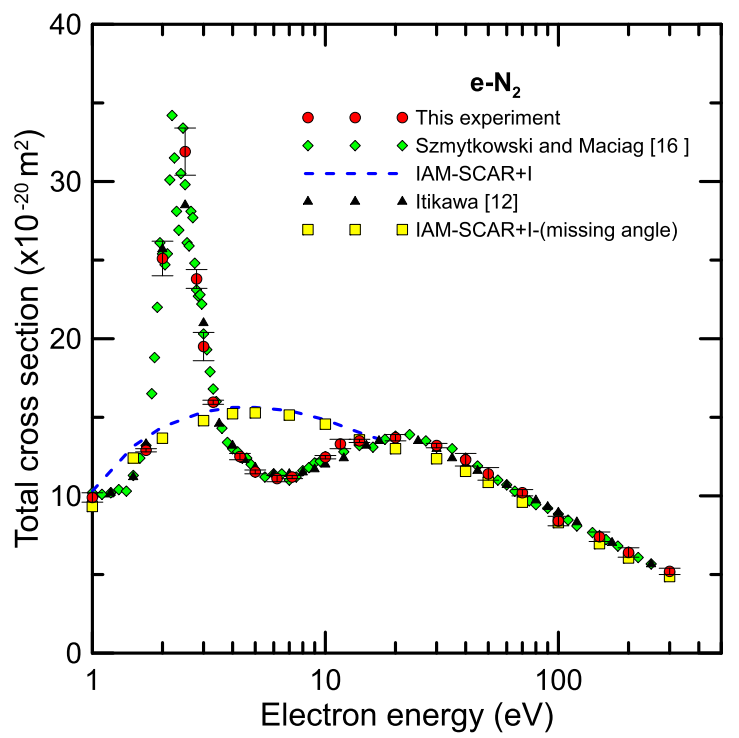

FIG. 7. Total electron scattering cross sections from $\mathrm{N}_{2}$ as a function of the incident electron energy together with the data available in the literature ${ }^{12,16}$ and our IAM-SCAR+I method. its molecular orbital structure, below $15 \mathrm{eV}$ our theoretical method does not follow the TCS variations and resonances observed by Szmytkowski and Maciag. ${ }^{16}$ However, it is still valid to evaluate the relevance of the missing angles. To perform such an assessment, differential elastic cross sections have been integrated for each incident electron energy from the missing angle limit to $180^{\circ}$ minus the missing angle as indicated in Eq. (10). As shown in Fig. 7, the results of this integration lead to a calculated TCS slightly lower than the original calculation. The relative differences vary from $9.7 \%$ to $2.2 \%$ for incident energies ranging from 1 to $20 \mathrm{eV}$. Being a systematic error which always tends to lower the measured cross sections, this percentage should be added to the experimental values in order to obtain reliable experimental data values. If we compare the direct measurements with the experimental values from Szmytkowski and Maciag ${ }^{16}$ and the recommended values from Itikawa, ${ }^{12}$ there is an excellent agreement within $3 \%$ for energies out of the resonance range $(2-3 \mathrm{eV})$. In this range, maximum discrepancies of the order of $7 \%$ are obtained. Note that the observed resonance intensities are very sensitive to energy resolutions used which are different for the experimental data we are comparing here. The transmission experiments reported in Refs. 12 and 16 do not use magnetic confinement, so their missing angle effects are expected to be much lower than in the present case. These authors did not give enough information on their experimental conditions to evaluate this systematic error, but the excellent general agreement found for $\mathrm{N}_{2}$ allows us to conclude that the systematic uncertainties derived from the missing angles and scattering length determination are not significant in this case and we can establish a total uncertainty limit of the present measurement within $7 \%$. We should note here that any further molecular target studied with the present experimental system will require a detailed missing angle effect evaluation based on reliable theoretical DCS calculations and especially for molecules with permanent dipole moments. ${ }^{20}$ We can anticipate that in this type of targets, the elastic cross section is dipole driven showing an enhancement in the forward direction (typically below $10^{\circ}$ ) of the scattered electrons.

\section{CONCLUDING REMARKS}

The novel experimental setup described here allows us to perform electron beam transmission measurements providing reliable total cross section data for gaseous molecular targets. The magnetic field confinement of the electron beam ensures scattering measurements at low incident energies, where resonances may dominate. The main limitation of the system arises from the missing angles derived from the energy resolution limit. However, we have shown that by using our IAM-SCAR+I calculation procedure, the magnitude of the systematic errors induced by the missing angles can be properly evaluated.

\section{ACKNOWLEDGMENTS}

This study has partially been financed by the Spanish Ministry MINECO (Project No. FIS2016-80440). K.K., F.F.S., 
and P.L.V. acknowledge the Portuguese National Funding Agency FCT through Grant No. SFRH/BD/52536/2014 and Research Grant No. UID/FIS/00068/2013. This work was also supported by the Radiation Biology and Biophysics Doctoral Training Programme (RaBBiT, No. PD/00193/2010); No. UID/Multi/04378/2013 (UCIBIO).

${ }^{1}$ T. Murphy and C. M. Surko, Phys. Rev. A 46, 5696 (1992).

${ }^{2}$ S. J. Gilbert, C. Kurz, R. G. Greaves, and C. M. Surko, Appl. Phys. Lett. 70, 1944 (1997).

${ }^{3}$ J. Clarke, D. P. van der Werf, B. Griffiths, D. C. S. Beddows, M. Charlton, H. H. Telle, and P. R. Watkeys, Rev. Sci. Instrum. 77, 063302 (2006).

${ }^{4}$ D. B. Cassidy, S. H. M. Deng, R. G. Greaves, and A. P. Mills, Jr., Rev. Sci. Instrum. 77, 073106 (2006).

${ }^{5}$ J. P. Sullivan, A. Jones, P. Caradonna, C. Makochekanwa, and S. J. Buckman, Rev. Sci. Instrum. 79, 113105 (2008).

${ }^{6}$ J. P. Sullivan, J. Roberts, R. W. Weed, M. R. Went, D. S. Newman, and S. J. Buckman, Meas. Sci. Technol. 21, 085702 (2010).

${ }^{7}$ J. R. Danielson, D. H. E. Dubin, R. G. Greaves, and C. M. Surko, Rev. Mod. Phys. 87, 247 (2015).

${ }^{8}$ M. C. Fuss, A. G. Sanz, F. Blanco, J. C. Oller, P. Limão-Vieira, M. Brunger, and G. García, Phys. Rev. A 88, 042702 (2013).
${ }^{9}$ A. G. Sanz, M. C. Fuss, F. Blanco, J. D. Gorfinkiel, D. Almeida, F. Ferreira da Silva, P. Limão-Vieira, M. J. Brunger, and G. García, J. Chem. Phys. 139, 184310 (2013).

${ }^{10}$ A. I. Lozano, K. Krupa, F. Ferreira da Silva, P. Limão-Vieira, F. Blanco, A. Muñoz, D. B. Jones, M. J. Brunger, and G. García, Eur. Phys. J. D 71, 226 (2017).

${ }^{11}$ F. Blanco and G. García, Chem. Phys. Lett. 635, 321 (2015).

${ }^{12}$ Y. Itikawa, J. Phys. Chem. Ref. Data 35, 31 (2006).

${ }^{13}$ S. J. Gilbert, R. G. Greaves, and C. M. Surko, Phys. Rev. Lett. 82, 5032 (1999).

${ }^{14}$ J. P. Sullivan, S. J. Gilbert, J. P. Marler, R. G. Greaves, S. J. Buckman, and C. M. Surko, Phys. Rev. A 66, 042708 (2002).

${ }^{15}$ W. Sun, M. A. Morrison, W. A. Isaacs, W. K. Trail, D. T. Alle, R. J. Gulley, M. J. Brennan, and S. J. Buckman, Phys. Rev. A 52, 1229 (1995).

${ }^{16}$ C. Szmytkowski and K. Maciag, Phys. Scr. 54, 271 (1996).

${ }^{17}$ F. Blanco and G. García, Phys. Rev. A 67, 022701 (2003).

${ }^{18}$ F. Blanco and G. García, Phys. Lett. A 317, 458 (2003).

${ }^{19}$ F. Blanco, L. Ellis-Gibbings, and G. García, Chem. Phys. Lett. 645, 71 (2016).

${ }^{20}$ W. Tattersall, L. Chiari, J. R. Machacek, E. Anderson, R. D. White, M. J. Brunger, S. J. Buckman, G. García, F. Blanco, and J. P. Sullivan, J. Chem. Phys. 140, 044320 (2014). 